\title{
Impact of sodium laser guide star fratricide on multi-conjugate adaptive optics systems
}

\author{
Lianqi Wang, * Angel Otarola, and Brent Ellerbroek \\ Thirty Meter Telescope Project, 2632 E. Washington Blvd., Pasadena, California 91107, USA \\ *Corresponding author: lianqiw@tmt.org
}

Received April 12, 2010; accepted June 1, 2010;

posted June 7, 2010 (Doc. ID 126821); published June 23, 2010

\begin{abstract}
Laser beams projected from the ground to form sodium layer laser guide stars (LGSs) for adaptive optics (AO) systems experience scattering and absorption that reduce their intensity as they propagate upward through the atmosphere. Some fraction of the scattered light will be collected by the other wavefront sensors and causes additional background in parts of the pupil. This cross-talk between different LGS wavefront sensors is referred to as the fratricide effect. In this paper we quantify the magnitude of four different sources of scattering/ absorption and backscattering, and we evaluate their impact on performance with various zenith angles and turbulence profiles for one particular AO system. The resulting wavefront error for the Thirty Meter Telescope (TMT) multi-conjugate AO (MCAO) system, NFIRAOS, is on the order of 5 to $20 \mathrm{~nm}$ RMS, provided that the mean background from the fratricide effect can be calibrated and subtracted with an accuracy of $80 \%$. We also present the impact on system performance of momentary variations in LGS signal levels due to variations in cirrus absorption or laser power, and we show that this affects the performance more than does an equal variation in the level of the fratricide. (C) 2010 Optical Society of America
\end{abstract}

OCIS codes: $010.1080,010.7350$.

\section{INTRODUCTION}

Current [1-3] and next-generation [4,5] ground-based astronomical telescopes rely upon laser guide star (LGS) adaptive optics (AO) systems to mitigate the image blurring due to atmospheric turbulence to achieve diffractionlimited resolution. Sodium LGS multi-conjugate AO (MCAO) is a popular method for mitigating the image blurring in a modest wide field of view (tens of arcsec to 1-2 arcmin) by measuring the turbulence along several different directions and reconstructing a 3-D profile of the turbulence.

When the laser beam passes through the atmosphere, it will experience scattering and absorption that reduce its intensity as it propagates through the medium. Some fraction of the light lost due to scattering will be back scattered in the direction of the transmitter. In a LGS MCAO system such as the Thirty Meter Telescope (TMT) narrow-field infrared AO system (NFIRAOS), a fraction of the back-scattered light from each of the laser beams ends up being collected by WFSs observing the other LGSs. This effect is generally referred to as the fratricide effect. The fratricide effect is prominent mostly for MCAO systems that launch all the laser beams from near the center of the primary mirror (e.g., behind the secondary mirror). The effect is also stronger at higher zenith angles.

The important sources of scattering and absorption in the atmosphere are Rayleigh scattering, ozone Chappiusband absorption, aerosol, and cirrus cloud scattering. The scattered (or re-emitted) light is usually not isotropic. The so-called phase function describes the angular distribution of the scattered light. When the scattering particle is much smaller than the wavelength of the incident photon (i.e., in the regime of Rayleigh scattering), the phase function is symmetric in the forward and backward directions. On the other hand, when the scattering particle is similar to or larger than the wavelength of the incident photon (i.e., in the regime of Mie scattering), the forward scattering is dominant. The aerosol and cirrus cloud scattering fall into this latter category and therefore does not contribute significantly to the fratricide effect.

In this paper, we describe the methods for quantifying the sources of scattering and/or absorption, as well as the back-scattered light that causes the fratricide effect. We will present the results on the impact of fratricide on the performance of MCAO systems at different zenith angles and seeing conditions. We have found that, for the TMT NFIRAOS system and the measured Mauna Kea 13N (MK13N) 25th and 50th percentile seeing profiles, with $80 \%$-level calibration of the fratricide effect (assumes $20 \%$ variation within the period of updating pixel-processing routines to account for the background), the incremental wavefront error is less than $10 \mathrm{~nm}$ RMS for zenith angles up to $45 \mathrm{deg}$. We also present the results on the impact on system performance due to momentary reduction of LGS signal levels due to increased absorption. A $20 \%$ reduction in the laser return signal level as collected by the wavefront sensors has a greater impact on the system performance than does the fratricide effect for most cases.

The remainder of the paper is organized as follows. Section 2 describes the sources of the scattering/absorption and presents the calculation of their volume scattering/ absorption coefficients and optical depth. Section 3 de- 
scribes the modeling of the wavefront sensor images due to the fratricide effect. Section 4 presents the results.

\section{SOURCES OF FRATRICIDE AND THEIR OPTICAL DEPTH}

In order to quantify the fratricide effect, we first need to compute the volume scattering/absorption coefficients and corresponding optical depth for the various atmospheric constituents that are relevant for this study.

\section{A. Rayleigh Scattering}

The total Rayleigh-scattering cross-section per molecule, $\sigma$, is given by the following formula [6-9]:

$$
\sigma(\lambda)=\frac{24 \pi^{3}\left(n_{s}^{2}-1\right)^{2}}{\lambda^{4} N_{s}^{2}\left(n_{s}^{2}+2\right)^{2}}\left(\frac{6+3 \rho_{n}}{6-7 \rho_{n}}\right),
$$

where $N_{s}$ and $n_{s}$ are the molecular number density and the refractive index of standard air, respectively. Standard air is defined as dry air containing $0.03 \% \mathrm{CO}_{2}$ by volume, at normal pressure $760 \mathrm{~mm} \mathrm{Hg}$ or $101325 \mathrm{~Pa}$, and a temperature of $15^{\circ} \mathrm{C}$. According to Lorenz-Lorentz and Clausius-Mossotti theory, the quantity $\left(n_{s}^{2}-1\right) /\left(n_{s}^{2}+2\right)$ is proportional to the molecular number density $N_{s}$, and therefore $\sigma(\lambda)$ is actually independent of both $n_{s}$ and $N_{s}$, which makes sense. The last term,

$$
F_{k}=\frac{6+3 \rho_{n}}{6-7 \rho_{n}}
$$

is the King correction factor, computed from the depolarization factor $\rho_{n}$, which is a function of $\lambda$. This term accounts for the anisotropy of the air molecules.

The amount of scattering for a volume of gas in the atmosphere is characterized by the total Rayleigh volumescattering coefficient $\beta$,

$$
\beta(\lambda, h)=N(h) \sigma(\lambda),
$$

where $N(h)$ is the number density as a function of altitude $h$. The light loss during radiation transfer is

$$
\frac{\mathrm{d} I}{I}=-\beta(\lambda, h) \mathrm{d} h
$$

which gives

$$
I(h)=I\left(h_{0}\right) \exp \left[-\int_{h_{0}}^{h} \beta(\lambda, h) \mathrm{d} h\right] .
$$

The value of the integral is conveniently called the optical depth $\tau$,

$$
\tau\left(\lambda, h_{0}\right)=\int_{h_{0}}^{\infty} \beta(\lambda, h) \mathrm{d} h
$$

for light passing from free space and arriving at altitude $h_{0}$. For the laser beacon launched from the observatory, we have

$$
\tau^{\prime}\left(\lambda, h_{0}\right)=\int_{h_{t}}^{h_{0}} \beta(\lambda, h) \mathrm{d} h,
$$

where $h_{t}$ is the altitude of the transmitter.

The angular distribution of the scattered light is described by the Rayleigh phase function $P_{\text {ray. For un- }}$ polarized incident radiation, $P_{\text {ray }}$ is simply

$$
P_{\text {ray }}(\theta)=\frac{3}{4}\left(1+\cos ^{2} \theta\right) \text {. }
$$

However, because molecular anisotropy also affects the angular distribution of Rayleigh-scattered light, a more accurate formula is given by

$$
P_{\text {ray }}(\theta)=\frac{3}{4(1+2 \gamma)}\left[(1+3 \gamma)+(1-\gamma) \cos ^{2} \theta\right],
$$

with

$$
\gamma=\frac{\rho_{n}}{2-\rho_{n}}
$$

The angular volume-scattering coefficient $\beta(\lambda, h, \theta)$ is then

$$
\beta(\lambda, h, \theta)=\beta(\lambda, h) \frac{P_{\text {ray }}(\theta, \lambda)}{4 \pi} .
$$

For the sodium laser wavelength at $589 \mathrm{~nm}, \gamma=1.395$ $\times 10^{-2}$; therefore

$$
P_{\text {ray }}(\theta)=0.73 \times\left(1.0418+0.986 \cos ^{2} \theta\right) .
$$

1. Standard Air at $15^{\circ} \mathrm{C}$

For standard air, we have

$$
\beta_{s}(\lambda)=N_{s} \sigma(\lambda) .
$$

The value of $\sigma(\lambda)$ is adopted from [9]:

$$
\sigma(\lambda=589 \mathrm{~nm})=3.304 \times 10^{-27} \mathrm{~cm}^{2} .
$$

The molecular number density for standard air is

$$
N_{s}=2.54743 \times 10^{19} \mathrm{~cm}^{-3}
$$

therefore

$$
\beta_{s}(\lambda=589 \mathrm{~nm})=8.406519 \times 10^{-3} \mathrm{~km}^{-1} .
$$

\section{Real Air Using the U.S. Standard Atmosphere Model}

The U.S. standard atmosphere model [10] treats the air as dry, ideal gas. The Rayleigh volume-scattering coefficient for the standard atmosphere model is related to the standard $\beta_{s}$ as

$$
\beta(\lambda, h)=\beta_{s}(\lambda) \frac{N(h)}{N_{s}}=\beta_{s}(\lambda) \frac{P(h)}{P_{s}} \frac{T_{s}}{T(h)},
$$

where $P_{s}$ and $T_{s}$ represent the reference pressure and temperature, $P_{s}=101325 \mathrm{~Pa}$, and $T_{s}=288.15 \mathrm{~K}$.

The U.S. standard air model describes the annually averaged atmospheric temperature using seven layers. For each layer, the temperature changes approximately lin- 
Table 1. U.S. Standard Air Model

\begin{tabular}{|c|c|c|c|c|c|}
\hline $\begin{array}{c}\text { Layer } \\
i\end{array}$ & $\begin{array}{l}\text { Base } \\
\text { Altitude } h_{i} \\
\quad(\mathrm{Km})\end{array}$ & $\begin{array}{c}\text { Standard } \\
\text { Temperature } \\
T_{i}(\mathrm{~K})\end{array}$ & $\begin{array}{c}\text { Temperature } \\
\text { Lapse Rate } \Gamma_{i} \\
(\mathrm{~K} / \mathrm{km})\end{array}$ & $\begin{array}{c}\text { Static } \\
\text { Pressure } P_{i} \\
(\mathrm{~Pa})\end{array}$ & $\begin{array}{c}\text { Back-Scatter } \\
\text { Coefficient } \\
\text { Scaling } \frac{P_{i}}{P_{s}} \frac{T_{s}}{T_{i}}\end{array}$ \\
\hline 0 & 0 & 288.15 & -6.5 & 101325 & 1.0000 \\
\hline 1 & 11 & 216.65 & 0.0 & 22632.1 & 0.2971 \\
\hline 2 & 20 & 216.65 & 1.0 & 5474.89 & 0.0719 \\
\hline 3 & 32 & 228.65 & 2.8 & 868.019 & 0.0108 \\
\hline 4 & 47 & 270.65 & 0.0 & 110.906 & 0.0012 \\
\hline 5 & 51 & 270.65 & 2.8 & 66.9389 & 0.0007 \\
\hline 6 & 71 & 214.65 & -2.0 & 3.95642 & 0.0001 \\
\hline
\end{tabular}

early with altitude with a lapse rate. Table 1 lists the temperature and pressure properties of each layer. We use this standard air model to approximate the air above Mauna Kea. The error in the computed optical depth caused by this approximation is within $1 \%$ in comparison with results by others and can be ignored for our purposes.

Let $h_{i}$ be the base altitude of layer $i$; the temperature in that layer is given by

$$
T=T_{i}+\Gamma_{i}\left(h-h_{i}\right),
$$

where $T_{i}$ is the standard temperature (base) at the bottom of that layer and $L_{i}$ is the lapse rate as previously mentioned. From the ideal gas law, $P=\rho R T$. From the hydrostatic equilibrium we have

$$
\frac{\mathrm{d} P}{\mathrm{~d} h}=-g \rho=-g \frac{P}{R T}=-\frac{g}{R} \frac{P}{T_{i}+\Gamma_{i}\left(h-h_{i}\right)},
$$

or

$$
\frac{\mathrm{d} P}{P}=-\frac{g}{R} \frac{\mathrm{d} h}{T_{i}+\Gamma_{i}\left(h-h_{i}\right)} .
$$

Therefore,
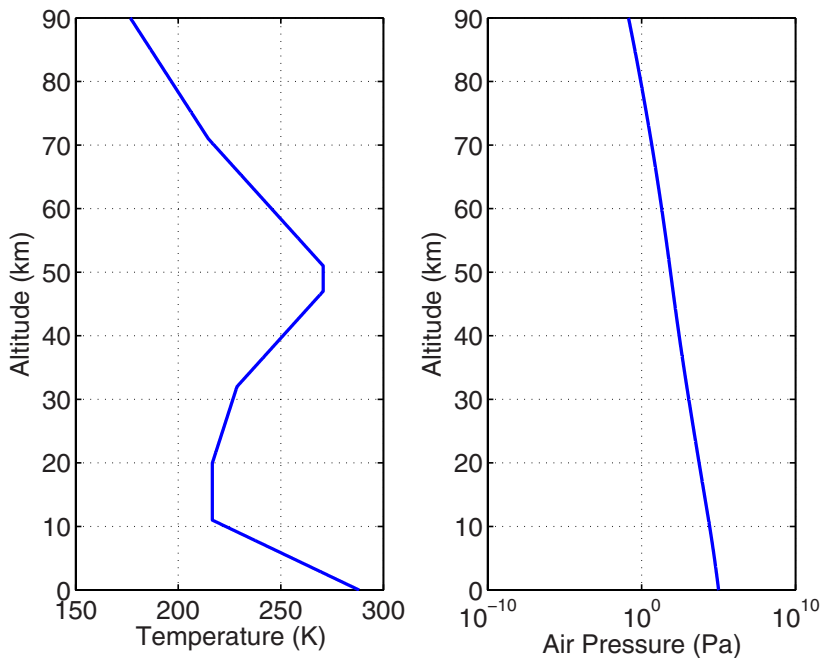

Fig. 1. (Color online) Temperature and pressure distribution of the U.S. standard atmosphere.

$$
\frac{P}{P_{i}}=\left\{\begin{array}{ll}
\left(1+\frac{\Gamma_{i}\left(h-h_{i}\right)}{T_{i}}\right)^{-g / R \Gamma_{i}}=\left(\frac{T}{T_{i}}\right)^{-g / R \Gamma_{i}}, & \Gamma_{i} \neq 0 \\
\exp \left[-\frac{g\left(h-h_{i}\right)}{R T_{i}}\right], & \Gamma_{i}=0
\end{array} .\right.
$$

The constants used here are $g=9.80665 \mathrm{~ms}^{-2}, R$ $=8.31432 \mathrm{~J} /(\mathrm{mole} \cdot \mathrm{K}) / 0.0289644 \mathrm{~kg} / \mathrm{mole}$

$=287.053 \mathrm{~J} /(\mathrm{kg} \cdot \mathrm{K})$. The change of $g$ is neglected since the altitude is small compared with the radius of the Earth.

Based on the above information, we are ready to compute the Rayleigh volume-scattering coefficient and the optical depth at any altitude. Figure 1 shows the vertical profile of air temperature and barometric pressure in the altitude range of interest. Figure 2 plots the Rayleigh volume-scattering coefficients and up-link optical depth as a function of the distance above the TMT MK13N site at an altitude of $4050 \mathrm{~m}$. The total Rayleigh optical depth from MK13N up to free space is about 0.0443 .

A direct fit to the Rayleigh volume-scattering coefficients for the standard atmosphere model at $0.589 \mu \mathrm{m}$ above the TMT MK13N site gives
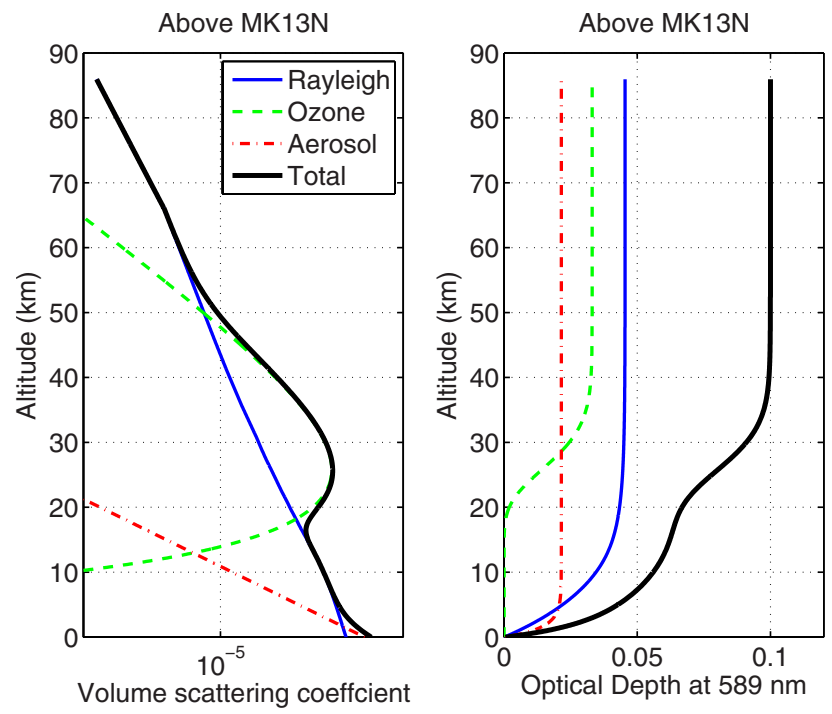

Fig. 2. (Color online) Volume-scattering coefficient and optical depth for Rayleigh, ozone Chappius band, and aerosol scattering computed above the TMT MK13N site. 


$$
\beta(h)=0.00599 \exp (-h / 6.9491),
$$

where $h$ is expressed in kilometer. The scale height is $6.95 \mathrm{~km}$. The precise values are used during the computation, instead of this fit.

\section{B. Chappius Band of Ozone Absorption}

The Chappius band of ozone is the wavelength band of light absorbed by ozone in the visible region, approximately $380-750 \mathrm{~nm}$. (The ultraviolet absorption region of ozone is called the Hartley-Huggins band.)

The vertical distribution of the numerical density of ozone [11] can be approximated by

$$
N_{\mathrm{O}_{3}}(h)=5.7 \times 10^{18} \exp \left(-\{[\log (h)-3.25] / 0.26\}^{2}\right) \mathrm{m}^{-3},
$$

with $h$ in kilometers. The absorption cross section [12] is

$$
\sigma_{\mathrm{O}_{3}}=4.8 \times 10^{-25} \mathrm{~m}^{2} .
$$

The volume absorption coefficient and the optical depth can be calculated in the same way as the Rayleigh back scattering. Figure 2 shows the result. The total optical depth for ozone Chappius band absorption above MK13N is about 0.0331 .

The stratospheric ozone does not play an important role in the overall molecular back scattering because the stratosphere is in a state of local thermodynamic equilibrium, which implies that the time between molecular collisions is shorter than that of spontaneous emission of radiation. Therefore we just set the phase function to zero.

\section{Aerosol Scattering}

Atmospheric aerosols are very diverse. They include tiny grains of mineral dust stirred up from the ground, particles of salt left when droplets of sea spray evaporate, bacteria, pollen grains, mold spores, other biosol particles, etc. Most of these are produced at or near ground level and are washed out of the atmosphere by condensation of cloud droplets on them, followed by precipitation. The aerosols all tend to be concentrated in the lowest part of the atmosphere. An exponential distribution with scale height of about $1.5 \mathrm{~km}$ is a rough approximation to their vertical distribution [13].

At the Gemini website [14], the overall extinction for photometric air is roughly $m=0.11 \mathrm{mag} /$ airmass at $589 \mathrm{~nm}$ for Mauna Kea. The conversion between extinction expressed in magnitude $m$ and optical depth $\tau$ can be computed by

$$
e^{-\tau}=10^{-m / 2.5},
$$

or

$$
\tau=0.9210 \mathrm{~m} .
$$

Therefore, the optical depth at Mauna Kea is about 0.10. Subtracting the optical depth of Rayleigh scattering and ozone Chappius band absorption, the optical depth for aerosol is about 0.0226 , which is in agreement with the measurements in [15].

The phase function of aerosol scattering is very different from that of Rayleigh scattering, since the aerosol particle sizes that have strong scattering are generally as-
Table 2. Total Optical Depth Contributions from Rayleigh, Ozone Chappius Band, Aerosol Scattering, and Cirrus Clouds

\begin{tabular}{cc}
\hline Source & Optical Depth \\
\hline Rayleigh & 0.0443 \\
Ozone & 0.0331 \\
Aerosol & 0.0226 \\
Cirrus & 0.2200 \\
\hline
\end{tabular}

sumed to be close to the wavelength of scattered light. (This assumption will be verified when data become available.) The wavelength dependence of aerosol scattering is almost inversely proportional to the wavelength $\left(\alpha \lambda^{-1.2}\right.$ power law is often used) [13]. The fraction of backscattered light $P_{\text {Aerosol }}(\pi) / 4 \pi$ in units of per solid angle is $0.0035 \mathrm{str}^{-1}$, roughly 40 times smaller than $0.12 \mathrm{str}^{-1}$ of the Rayleigh scatter.

\section{Cirrus Clouds}

Cirrus clouds are ice clouds that form at temperatures below $-40^{\circ} \mathrm{C}$ [16]. Near the tropics, as is Mauna Kea, cirrus happens above $8 \mathrm{~km}$, with a mean altitude of $13.5 \mathrm{~km}$ above sea level. The mean thickness is $1.6 \mathrm{~km}$. The vertical distribution can be approximated by a Gaussian. The average value for the total optical depth is about 0.22 . For a reference, the cirrus cloud frequency over the Hawaii region in the period October/November 2003, obtained from the ICESat/GLASS and MODIS/Terra missions is on the order of $25 \%$ [see Figs. (3a) and 3(b) in [16]]. The sizes of cirrus ice particles are larger than or similar to the wavelength of visible light, so the fraction of back scatter is similar to that of aerosol scatter.

\section{E. Summary}

Figure 2 plots the volume scattering/absorption coefficients and up-link optical depth of the four effects excluding cirrus as a function of the distance above the TMT $\mathrm{Mk} 13 \mathrm{~N}$ site at an altitude of $4050 \mathrm{~m}$. Table 2 summarizes the contributions of these four sources to optical depth. Temporal variations in cirrus and aerosols will affect atmospheric transmittance much more than back scatter will, for typical particle size distributions.

\section{FRATRICIDE MODELING}

Here we are going to ignore the diffraction effects and will model the fratricide using geometric optics. The light intensity in the sky on a horizontal plane at range $h$ above the telescope is

$$
\begin{aligned}
I(\mathbf{x}, h)= & e^{-\tau(h)} \sum_{i=1}^{N} I_{i}(\mathbf{x}, h)=e^{-\tau(h)} \sum_{i=1}^{N} \rho_{i} L_{i}(\mathbf{x}), \\
L_{i}(\mathbf{x})= & \operatorname{circ}\left[\frac{\mathbf{x}-\left(\mathbf{r}_{i}+h \boldsymbol{\theta}_{i}\right)}{R_{L L T}}\right] \\
& \times \exp \left[-\frac{\left(\mathbf{x}-\left(\mathbf{r}_{i}+h \boldsymbol{\theta}_{i}\right)\right)^{2}}{2 \sigma^{2}}\right],
\end{aligned}
$$

where $\mathbf{x}$ is the coordinate on the horizontal plane; $\mathbf{r}_{i}, \theta_{i}$ 
are the location and the beam direction of the laser launch telescope for WFS $i$, respectively, and therefore $\mathbf{r}_{i}+h \theta_{i}$ is the center of the beam on the plane at range $h$; $R_{L L T}$ is the radius of the clear aperture of the laser launch telescope; and, finally, $\sigma$ describes the width of the Gaussian laser beam. $N$ is the number of LGS WFSs $(N=6$ for NFIRAOS), $L_{i}(\mathbf{x})$ is the beam intensity function for the Laser launch telescope for WFS $i$ as further described in Subsection 7.A, and, finally, $\rho$ is the on-axis intensity of the laser beam at the ground level in units of power per unit area in units of $\mathrm{W} \mathrm{m}^{-2}$, computed from the laser power and the total transmittance of the uplink LGS facility optics. The intensity $I(\mathbf{r}, h)$ also has units of power per unit area. The function $\tau(h)$ is one-way up-link sky optical depth, which is related to the volume scattering/ absorption coefficient $\beta(h)$ by

$$
\tau(h)=\int_{h_{t}}^{h} \mathrm{~d} h^{\prime} \beta\left(h^{\prime}\right) .
$$

The scattered light intensity back toward the telescope is

$$
R(\mathbf{x}, h)=e^{-\tau(h)} \beta(h, \pi) I(\mathbf{x}, h),
$$

where $\beta(h, \pi)=\beta(h)[P(\pi) / 4 \pi]$ is the volume backscattering/absorption coefficient as described in Section 2, and the additional factor of $e^{-\tau(h)}$ is due to the light loss when the scattered light propagates back downward. The scattered light intensity $R(\mathbf{x}, \mathrm{h})$ has units of power per unit volume per unit solid angle, $\mathrm{W} \mathrm{m}^{-3} \mathrm{sr}^{-1}$.

The integrated intensity of the scattered light on LGS WFS $j$, subaperture $k$, pixel $l$, is then

$$
P_{j k l}=\gamma_{t} \int \mathrm{d} \boldsymbol{\phi} d_{j k l}(\boldsymbol{\phi}) \int \mathrm{d} \mathbf{x} S_{j k}(\mathbf{x}) \int \mathrm{d} h R(\mathbf{x}+h \boldsymbol{\phi}, h),
$$

where $\gamma_{t}$ is the total optical throughput of the telescope and the LGS path in the instrument, $d_{j k l}(\boldsymbol{\phi})$ is the detector pixel influence function [the field-of-view (FOV) function of the pixel], $S_{j k}(\mathbf{x})$ is the subaperture pupil function, and $\mathbf{x}$ is the pupil plane coordinate. We rewrite this equation as

$$
\begin{aligned}
P_{j k l}= & \gamma_{t} \int \mathrm{d} \boldsymbol{\phi} d_{j k}\left(\boldsymbol{\phi}-\boldsymbol{\phi}_{j k l}\right) \int \mathrm{d} \mathbf{x} S_{0}\left(\mathbf{x}-\mathbf{x}_{k}\right) \\
& \cdot \int \mathrm{d} h e^{-2 \tau(h)} \beta(h, \pi) \sum_{i=1}^{N} \rho_{i} L_{i}(\mathbf{x}+h \boldsymbol{\phi}) \\
= & \gamma_{t} \sum_{i=1}^{N} \rho_{i} \int \mathrm{d} h e^{-2 \tau(h)} \beta(h, \pi) \\
& \cdot \int \mathrm{d} \boldsymbol{\phi} d_{j k}(\boldsymbol{\phi}) \int \mathrm{d} \mathbf{x} S_{0}(\mathbf{x}) L_{i}\left(\mathbf{x}+\mathbf{x}_{k}+h \boldsymbol{\phi}+h \boldsymbol{\phi}_{j k l}\right), \\
& \mathbf{r}_{j}-\mathbf{x}_{k} \\
\boldsymbol{\phi}_{j k l}= & \boldsymbol{\phi}_{l},
\end{aligned}
$$

where $\boldsymbol{\phi}_{j k l}$ is the center of the FoV for pixel $l$ in subaperture $k$ for WFS $j, \mathbf{r}_{j}$ is the location of the guide star for WFS $j$ on sky, $H$ is the height of guide star, and $\phi_{l}$ is the angular offset of pixel $l$ in its subaperture (expressed in the global $\mathrm{x}-\mathrm{y}$ coordinate system). The transmittance $e^{-2 \tau(h)}$ comes from the upward and downward light loss. The pixel function $d_{j k}$ defines the boundary of the pixels in subaperture $k$ of WFS $j$ for the polar coordinate CCD. $S_{0}(\mathbf{x})$ is the rectangular function defining the subaperture.

Let's treat the final line of Eq. (32) in detail. Define $P_{i j k l}(h)$ as

$$
P_{i j k l}(h)=\gamma_{t} \int \mathrm{d} \boldsymbol{\phi} d_{j k}(\boldsymbol{\phi}) \int \mathrm{d} \mathbf{x} S_{0}(\mathbf{x}) L_{i}\left(\mathbf{x}+\mathbf{x}_{k}+h \boldsymbol{\phi}+h \boldsymbol{\phi}_{j k l}\right) .
$$

This equation contains two convolutions over $\boldsymbol{\phi}$ and $\mathbf{x}$. We rewrite the integral as

$$
\begin{aligned}
P_{i j k l}(h)= & \gamma_{t} \int \mathrm{d} \boldsymbol{\phi} d_{j k}(\boldsymbol{\phi}) \int \mathrm{d} \boldsymbol{\kappa} \hat{S}_{0}(\boldsymbol{\kappa}) \\
& \times \int \mathrm{d} \mathbf{x} e^{2 \pi i \boldsymbol{\kappa} \cdot \mathbf{x}} L_{i}\left(\mathbf{x}+h \boldsymbol{\phi}+\mathbf{x}_{k}+h \boldsymbol{\phi}_{j k l}\right) \\
= & \gamma_{t} \int \mathrm{d} \boldsymbol{\kappa} \hat{S}_{0}(\boldsymbol{\kappa}) \hat{L}_{i}(-\boldsymbol{\kappa}) \int \mathrm{d} \boldsymbol{\phi} d_{j k}(\boldsymbol{\phi}) e^{-2 \pi i \boldsymbol{\kappa}(h \boldsymbol{\phi})} \\
& \times \exp \left[-2 \pi i \boldsymbol{\kappa} \cdot\left(\mathbf{x}_{k}+h \boldsymbol{\phi}_{j k l}\right)\right] \\
= & \gamma_{t} \mathcal{F}^{-1}\left[\hat{S}_{0}(\boldsymbol{\kappa}) \hat{L}_{i}(-\boldsymbol{\kappa}) \hat{d}_{j k}(h \boldsymbol{\kappa})\right]\left(-\mathbf{x}_{k}-h \boldsymbol{\phi}_{j k l}\right) .
\end{aligned}
$$

This is the inverse Fourier transform of the product of the Fourier transforms of (i) subaperture function $S_{0}$, (ii) LLT beam amplitude function $L_{i}$, and (iii) detector pixel influence function $d_{j k}$, evaluated at locations determined by the subaperture position and the pixel line of sight.

In Appendix A, we describe the computation of the Fourier transform of LLT pupil amplitude function $L_{i}$, subaperture function $S_{0}$, and detector pixel influence function $d_{j k}$. Substituting those results, (assuming an untruncated Gaussian laser beam), we get

$$
\begin{aligned}
P_{i j k l}(h)= & \gamma_{t} \mathcal{F}^{-1}\left\{2 \pi \sigma^{2} \exp \left[-2 \sigma^{2} \pi^{2}|\boldsymbol{\kappa}|^{2}\right] d^{2} \operatorname{sinc}\left(k_{x} d\right) \operatorname{sinc}\left(k_{y} d\right)\right. \\
& \cdot \Delta^{2} \operatorname{sinc}\left[h\left(k_{x} \cos \theta+k_{y} \sin \theta\right) \Delta\right] \operatorname{sinc}\left[h \left(k_{x} \sin \theta\right.\right. \\
& \left.\left.\left.-k_{y} \cos \theta\right) \Delta\right] \cdot \exp \left[-2 \pi^{2} \phi_{b l u r}^{2} h^{2}\left(k_{x}^{2}+k_{y}^{2}\right)\right]\right\}\left(\mathbf{r}_{i}+h \boldsymbol{\theta}_{i}\right. \\
& \left.-\mathbf{x}_{k}-h \boldsymbol{\phi}_{j k l}\right) .
\end{aligned}
$$

We can see that $P_{i j k l}(h)$ is dependent only on the separation between the center of the laser beam $\mathbf{r}_{i}+h \boldsymbol{\theta}_{i}$ and the mapped detector pixel location $\mathbf{x}_{k}+h \boldsymbol{\phi}_{j k l}$ at range $h$, which makes sense.

Figure 3 shows the LLT beam intensity function, the subaperture function, the detector pixel influence function (magnified 100 times), and the function $P_{i j k l}$ for two affected subapertures at two different ranges. Plots of the same type are plotted in the same scale for these two ranges. It is clear that the maximum intensity is independent of the range $h$, although the number of illuminated pixels and thus the total intensity varies roughly with $h^{-2}$. 

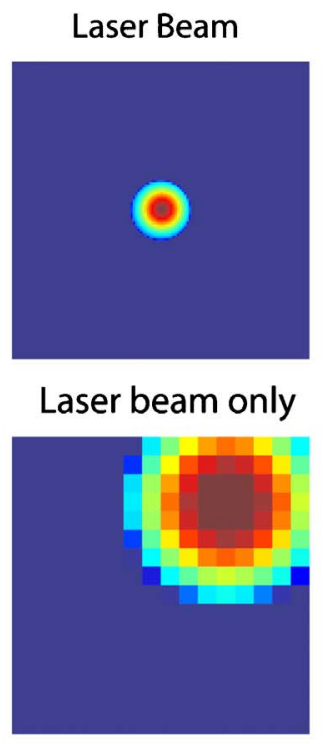

Laser Beam
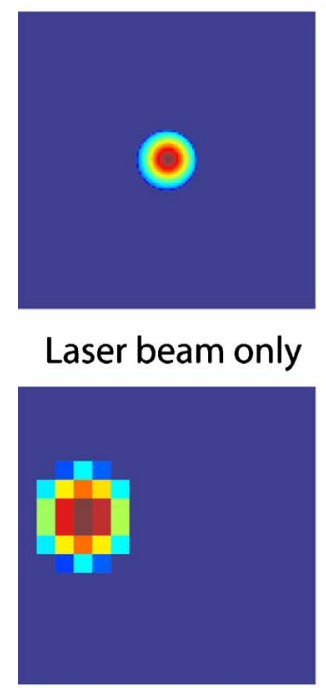

Subaperture

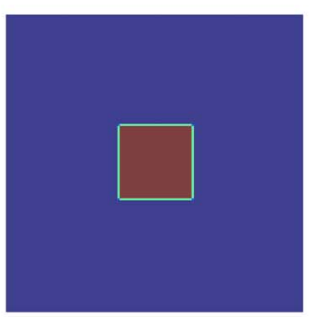

With subaperture

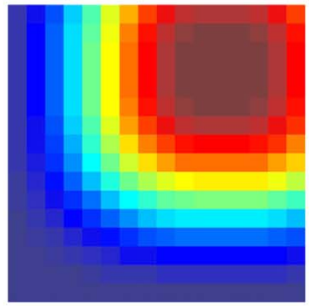

$\mathrm{h}=15 \mathrm{~km}$

Subaperture

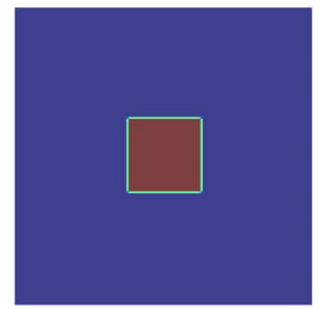

With subaperture

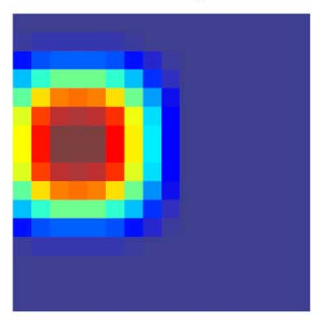

$\mathrm{h}=30 \mathrm{~km}$
Detector Pixel (100x)

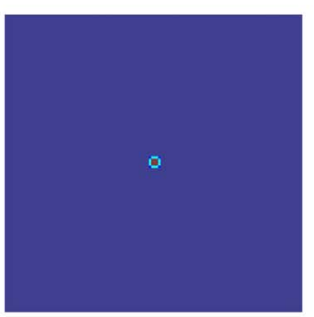

All included

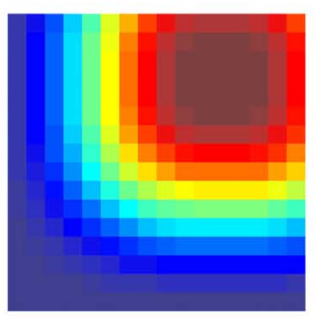

Detector Pixel (100x)

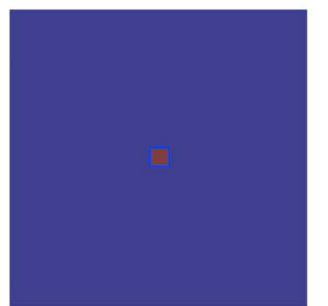

All included

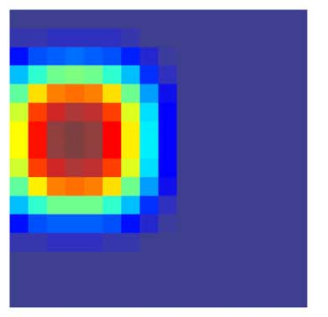

Fig. 3. (Color online) Plot of the LLT beam intensity function, the subaperture function, the detector pixel influence function (magnified 100 times), and the function $P_{i j k l}$ for a sample subaperture. The top two rows are for a $15 \mathrm{~km}$ layer, and the bottom two rows are for a $30 \mathrm{~km}$ layer.

It is worth noting that the Fourier transform of the subaperture function $\hat{S}_{0}$ is much narrower than the pixel influence function $\hat{d}_{j k}$ for our setup; thus we can ignore the radial orientation of the detector pixels in the polar coordinate CCD that will be used for NFIRAOS. As a consequence, we need to perform only one Fourier transform at each range $h$, which saves a lot of computation. The relative error caused by this simplification is on the order of $10^{-5}$.

\section{TIME-CONSTANT BACK-SCATTER VALUES}

\section{A. Parameter Summary}

We are assuming constant back-scatter patterns that are independent of time. This assumption is valid as long as the variations have a time scale of minutes or more, because the LGS pixel processing algorithm parameters are updated at a rate of about $0.1 \mathrm{~Hz}$. We are using the NFIRAOS asterism geometry, which is an order $60 \times 60$ dual-conjugate AO system with six LGS WFS arranged in a pentagon with $35^{\prime \prime}$ radius plus one more on axis. Each of the six LGSs has a laser power of $25 \mathrm{~W}$. They are all launched from a single LLT of diameter $0.4 \mathrm{~m}$ behind the TMT secondary mirror. The total transmittance of the uplink LGS facility is 0.75 . Each LGS WFS is employing a radial format CCD, where pixels in each subaperture are aligned along the direction of elongation to minimize the required number of pixels. Each pixel has $0.5^{\prime \prime} \times 0.5^{\prime \prime} \mathrm{FoV}$. The total transmittance of the telescope and the LGS path in the instrument is $0.81 \times 0.54=0.44$. The column density of the sodium atoms is assumed to be 3 

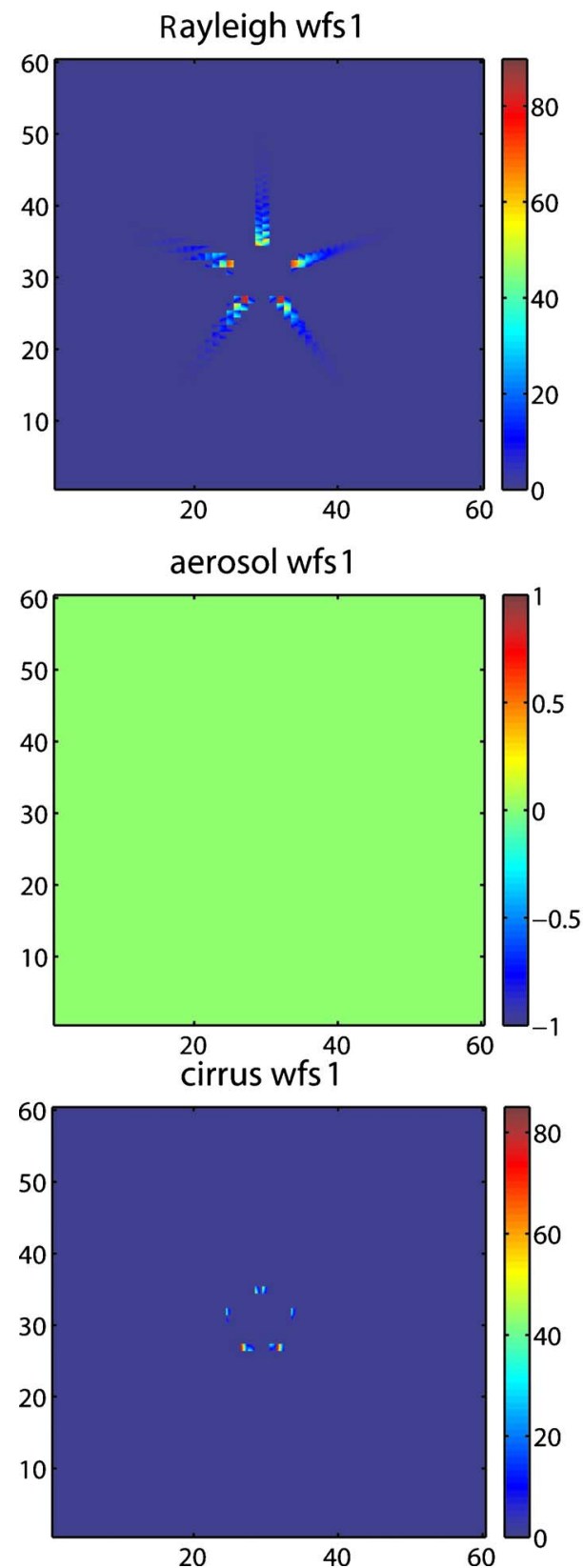

Fig. 4. (Color online) Back-scatter images for the NFIRAOS LGS WFS parameters in units of photons/frame/pixel.

$\times 10^{13}$ ions $/ \mathrm{m}^{2}$. The unsaturated coupling efficiency between the scattered photon counts per second per steradians of a single atom and the laser power density is 144 photons $/ \mathrm{s} /\left(\mathrm{W} / \mathrm{m}^{2}\right) /$ ion/sr. The equivalent transmittance due to imperfect laser beam quality is assumed to be 0.8 . Based on these parameters, we obtain a total of 900 photon detection events per subaperture per frame at $800 \mathrm{~Hz}$ at zenith for sodium LGS return. The coupling efficiency value of 144 is slightly larger than the TMT requirement of 130 , because we maintained the signal level as the TMT requirement but are using a smaller atmospheric transmittance of 0.78 instead of 0.84 than in the TMT requirement. Constrained matched filter [17] is used to precess the subaperture images to get wavefront gradient measurements. The constrained matched filter has better performance than centroid algorithms when noise
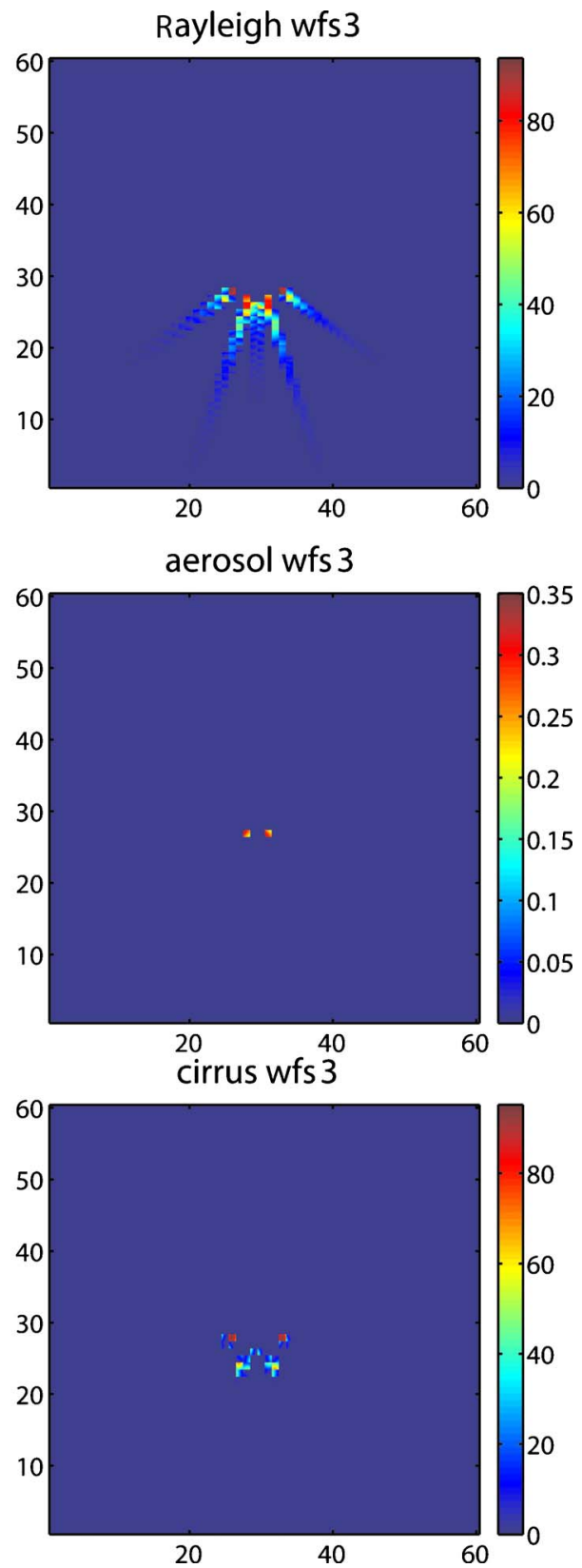

or bright background is present, especially on pixels where the weighting used by the matched filter is low.

\section{B. Back-Scatter Images}

Figure 4 shows the back-scatter images (excluding sodium layer return) for the four different sources except ozone, with telescope at zenith. The sub-image at the location of each subaperture is the subaperture image of size $15 \times 6$ along the radial/azimuthal direction. Only WFS 1 (on axis) and 3 (off axis) are shown. The other WFSs will be similar to the image of WFS 3 with a rotation according to the geometric orientation. The Rayleigh back scatter is the dominant term. The aerosol and cirrus scattering are concentrated in the inner subapertures because of low-altitude origin and weak back-scatter phase 

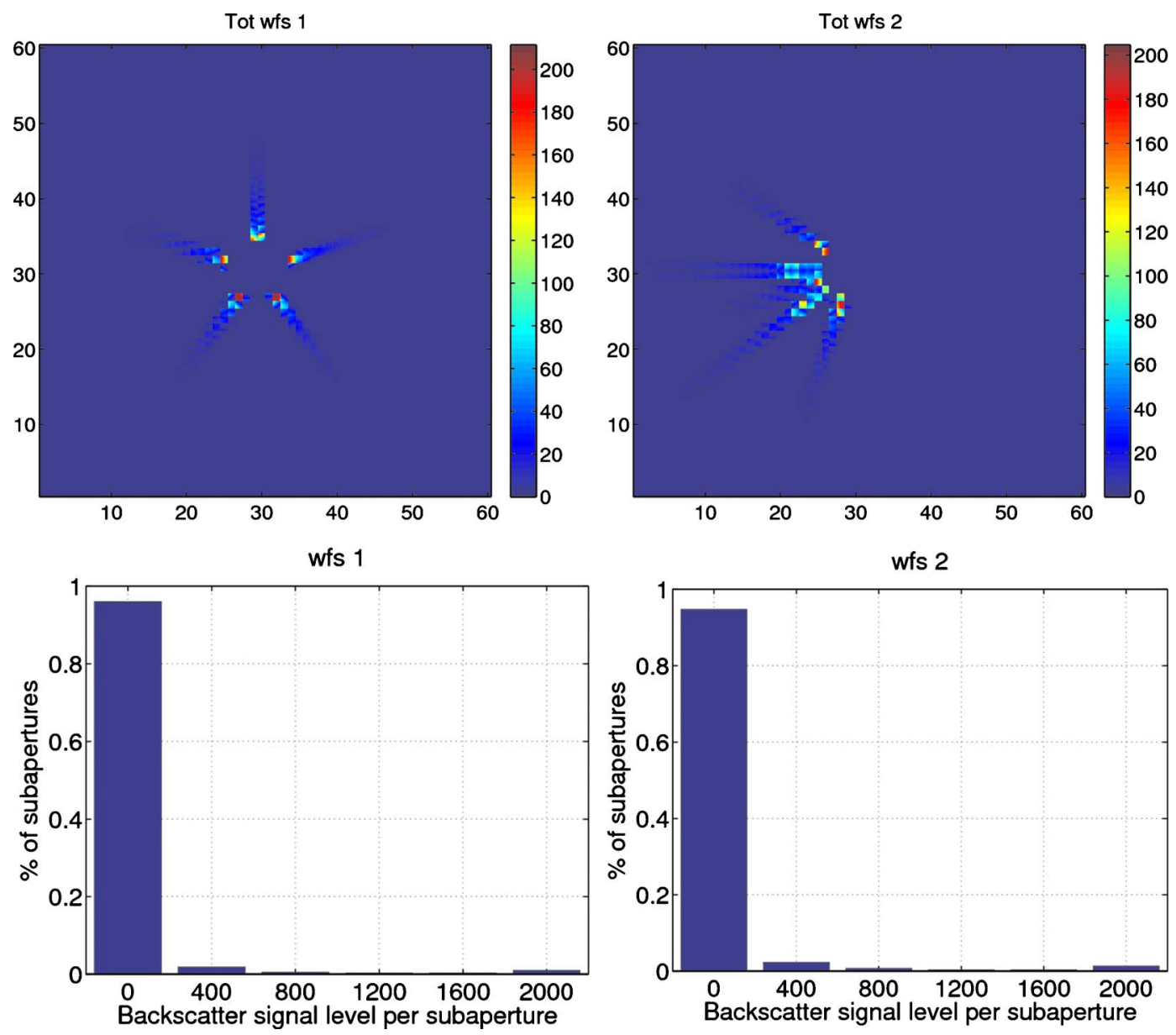

Fig. 5. (Color online) Total back-scatter image in units of photons/frame/pixel and their histogram.

function. Figure 5 shows the total back-scatter image of all four sources.

\section{Histograms of Signal Levels}

Figure 5 also shows the histogram of the total backscattered signal levels in each subaperture (excluding sodium layer return). The last bin contains the signal levels up to the maximum value. Recall that the LGS signal level will be about 900 photon detection events per subaperture for the median sodium column density and the specified laser power and sodium coupling efficiency. The number of pixels in each subaperture varies from $6 \times 6$ to
$15 \times 6$ in the real detector, but in simulations we are assuming that all subapertures have $16 \times 6$ pixels.

\section{IMPACT ON NFIRAOS PERFORMANCE}

The impact of the back scattering on NFIRAOS performance is evaluated with the TMT MK13N site $25 \%$ and $50 \% C_{n}^{2}$ profiles, and telescope zenith angles at $0,30,45$, and $60 \mathrm{deg}$. The results are shown in Table 3 . The constrained matched filter and the wavefront reconstructor are updated by taking into account the increased Poisson noise (and background if not fully calibrated), to account

Table 3. Impact of Fratricide on NFIRAOS Performance with MK13N 25\% and $50 \% C_{n}^{2}$ Profile $^{a}$

\begin{tabular}{|c|c|c|c|c|c|c|c|c|c|}
\hline \multicolumn{2}{|c|}{$C_{n}^{2}$ Profile } & \multicolumn{4}{|c|}{$25 \% \mathrm{Mk} 13 \mathrm{~N}$} & \multicolumn{4}{|c|}{$50 \% \mathrm{Mk} 13 \mathrm{~N}$} \\
\hline \multicolumn{2}{|c|}{ Zenith angle (deg) } & 0 & 30 & 45 & 60 & 0 & 30 & 45 & 60 \\
\hline No fratricide & LGS WFE (nm) & 94.6 & 106.1 & 130.2 & 193.7 & 118.0 & 131.5 & 159.7 & 234.0 \\
\hline Not calibrated & Incr. WFE (nm) & 9.8 & 15.5 & 29.2 & 84.3 & 11.8 & 19.7 & 38.9 & 116.8 \\
\hline Not calibrated & Ruined subaps. & $2.7 \%$ & $4.1 \%$ & $6.8 \%$ & $12.0 \%$ & $2.7 \%$ & $4.4 \%$ & $7.3 \%$ & $12.5 \%$ \\
\hline $80 \%$ calibrated & Incr. WFE (nm) & 3.6 & 4.0 & 7.8 & 20.5 & 1.4 & 4.7 & 10.3 & 30.6 \\
\hline $80 \%$ calibrated & Ruined subaps. & $0.4 \%$ & $0.7 \%$ & $1.4 \%$ & $4.3 \%$ & $0.4 \%$ & $0.7 \%$ & $1.5 \%$ & $4.6 \%$ \\
\hline $100 \%$ calibrated & Incr. WFE (nm) & 3.01 & 3.61 & 4.03 & 13.5 & 3.0 & 4.8 & 6.5 & 18.9 \\
\hline $100 \%$ calibrated & Ruined subaps. & $0.2 \%$ & $0.4 \%$ & $0.7 \%$ & $1.8 \%$ & $0.2 \%$ & $0.4 \%$ & $0.7 \%$ & $1.8 \%$ \\
\hline
\end{tabular}

${ }^{a}$ Four turbulence seeds with 500 time steps each are used to do the averaging. 
Table 4. NFIRAOS Performance Variations with Different Sodium Return Signal Levels for MK13N Profile ${ }^{a}$

\begin{tabular}{ccccccccc}
\hline$C_{n}^{2}$ Profile & \multicolumn{4}{c}{$25 \% \mathrm{Mk} 13 \mathrm{~N}$} & \multicolumn{3}{c}{$50 \% \mathrm{Mk} 13 \mathrm{~N}$} \\
\hline Zenith angle (deg) & 0 & 30 & 45 & 60 & 0 & 30 & 45 & 60 \\
WVE with no signal level change & 94.6 & 106.1 & 130.2 & 193.7 & 118.0 & 131.5 & 159.7 & 234.0 \\
Incremental WVE with 20\% reduction & 20.0 & 18.6 & 15.0 & 20.3 & 22.6 & 22.0 & 17.1 & 23.6 \\
Incremental WVE with 20\% increase & -5.4 & -6.1 & 5.4 & 25.7 & 3.4 & 6.5 & 9.6 & 33.2 \\
\hline
\end{tabular}

${ }^{a}$ The pixel processing matched filter is not aware of this change. Four turbulence seeds with 500 time steps each are used to do the averaging.

for the pixel background created by fratricide scattering. The following describes each case in the table in detail.

- Case "No fratricide": the baseline where no back scatter is considered.

- Case "Not calibrated": the worst case where the fratricide scattering pattern changes so quickly that it cannot be calibrated.

- Case "80\% calibrated": the most realistic case where the fratricide varies in the short term but can be calibrated at the $80 \%$ level.

- Case " $100 \%$ calibrated": the best case where the fratricide scattering pattern is very stable so that it can be completely calibrated during the matched-filter updating through LGS beam dithering. Only the photon noise is influencing the performance.

The incremental wavefront error is computed as the quadrature difference between each case and the baseline case where no fratricide effect is considered. For the baseline, the wavefront error in LGS controlled modes are shown in units of nanometers. For the other cases with fratricide effect included, the quadrature difference between the wavefront error in LGS controlled modes in that case and the baseline case is shown in units of nanometers. The percentages of ruined subapertures are also shown, which refer to those that have the measurement error (in milliarcsec) at least doubled due to the fratricide effect. For wavefront error numbers below $5 \mathrm{~nm}$, the numerical noise in simulation and averaging becomes important and the numbers should not be compared literally.

\section{A. Sensitivity to Change in Sodium Return Signal Level} The change in thickness of cirrus clouds or change in the output of the laser itself will cause the LGS sodium returns to vary. The change in cirrus and aerosol optical depth changes the total transmittance, but not the back scatter, appreciably. Table 4 shows the performance impact when the total LGS signal level changes by $\pm 20 \%$ from the nominal level for the MK13N 25\% and 50\% profiles at various zenith angles. We assume that the change of signal level is at high frequency so that the constrained matched filter is not able to track the change. At low zenith angles, a 20\% increase in the signal level has a smaller performance impact than reduction in the signal level, because the reduction in signal level causes not only a mismatch between the matched filter and the actual WFS images but also a reduction in the signal-to-noise ratio, while the increase in signal level improves the signalto-noise ratio. The impact on performance is manageable for changes within $\pm 20 \%$. When statistics on cirrus opti- cal depth are available, we will evaluate the performance impact using actual signal-level-variation statistics.

\section{CONCLUSION}

We have presented an analysis on fratricide effects, caused mainly by Rayleigh back scattering, and showed that the performance impact is less than $10 \mathrm{~nm}$ (quadratic increase in wavefront optical path difference error) for zenith angles up to $45 \mathrm{deg}$. The lower sensitivity to noise of the constrained matched filter used for the pixel process is a major advantage compared with other centroiding algorithms. The constrained matched filter puts most of its weights on pixels where the reference image (the time-averaged subaperture image) has large values and changes most as tip/tilt varies. The increased background due to Rayleigh back scattering on pixels where the weights are small does not appreciably affect the gradient measurements.

We also presented the simulation results on signal level variations and showed that a $20 \%$ reduction in signal level causes about $20 \mathrm{~nm}$ (quadratic increase) error, which is more severe than the fratricide effect with telescope zenith angles up to $45 \mathrm{deg}$ for the TMT NFIRAOS system.

\section{APPENDIX A}

\section{Fourier Transform of the LLT Pupil Amplitude Function $L_{i}$}

The Fourier transform pupil amplitude function for a Gaussian beam,

$$
L_{i}(\mathbf{x})=\exp \left[-\left(\mathbf{x}-\left(\mathbf{r}_{i}+h \boldsymbol{\theta}_{i}\right)\right) / 2 \sigma^{2}\right],
$$

can be computed analytically:

$$
\hat{L}_{i}(\boldsymbol{\kappa})=2 \pi \sigma^{2} \exp \left[-2 \sigma^{2} \pi^{2}|\boldsymbol{\kappa}|^{2}-2 \pi i \boldsymbol{\kappa} \cdot\left(\mathbf{r}_{i}+h \boldsymbol{\theta}_{i}\right)\right]
$$

but for the truncated beam, it has to be computed numerically.

2. Fourier Transform of the Subaperture Function $S_{0}$ The subaperture mask function is a rectangular function that defines the pupil of a subaperture, i.e.,

$$
S_{0}(\mathbf{x})=\sqcap(x / d) \sqcap(y / d) .
$$

The Fourier transform is simply

$$
\hat{S}_{0}(\boldsymbol{\kappa})=d^{2} \operatorname{sinc}\left(k_{x} d\right) \operatorname{sinc}\left(k_{y} d\right),
$$

where 


$$
\operatorname{sinc}(x) \equiv \frac{\sin (\pi x)}{\pi x} .
$$

\section{Fourier Transform of the Detector Pixel Influence Function}

We define the detector pixel influence function in a polar coordinate CCD in the local coordinate system with axes aligned along the radial and azimuthal directions. The pixel function can be modeled as a rectangular function that defines the edge of the pixel, convolved by a Gaussian blurring function that models the charge diffusion:

$$
\begin{aligned}
d_{j k}^{\prime}\left(\phi_{r a}\right)= & \sqcap\left(\phi_{r} / \Delta\right) \sqcap\left(\phi_{a} / \Delta\right) * \frac{1}{2 \pi \phi_{b l u r}^{2}} \\
& \times \exp \left[-\left(\phi_{r}^{2}+\phi_{a}^{2}\right) /\left(2 \phi_{b l u r}^{2}\right)\right] .
\end{aligned}
$$

The Fourier transform of this model is

$$
\hat{d}^{\prime}\left(u_{r a}\right)=\Delta^{2} \operatorname{sinc}\left(u_{r} \Delta\right) \operatorname{sinc}\left(u_{a} \Delta\right) \exp \left[-2 \pi^{2} \phi_{b l u r}^{2}\left(u_{r}^{2}+u_{a}^{2}\right)\right] .
$$

The relation between the coordinates in the local radialazimuthal coordinate system and the global Cartesian system is

$$
\phi_{r a}=R_{\alpha}^{T} \phi_{x y}
$$

where $R_{\alpha}$ is the transformation matrix for a rotation of angle $\alpha$ :

$$
R_{\alpha}=\left[\begin{array}{cc}
\cos \alpha & -\sin \alpha \\
\sin \alpha & \cos \alpha
\end{array}\right] .
$$

Therefore

$$
d_{j k}\left(\phi_{x y}\right)=d_{j k}^{\prime}\left(R_{\alpha}^{T} \phi_{x y}\right),
$$

and its Fourier transform is

$$
\begin{aligned}
\hat{d}_{j k}\left(u_{x y}\right) & =\int \mathrm{d}\left(R \phi_{r a}\right) d_{j k}^{\prime}\left(\phi_{r a}\right) \exp \left[-2 \pi i\left(R \phi_{r a}\right)^{T} u_{x y}\right] \\
& =\left|R_{\alpha}^{-1}\right| \int \mathrm{d} \phi_{r a} d_{j k}^{\prime}\left(\phi_{r a}\right) \exp \left[-2 \pi i \phi_{r a}^{T}\left(R^{T} u_{x y}\right)\right] \\
& =\hat{d}_{j k}^{\prime}\left(R_{\alpha}^{T} u_{x y}\right),
\end{aligned}
$$

or, more explicitly,

$$
\begin{aligned}
\hat{d}_{j k}\left(u_{x y}\right)= & \Delta^{2} \operatorname{sinc}\left[\left(u_{x} \cos \theta+u_{y} \sin \theta\right) \Delta\right] \operatorname{sinc}\left[\left(u_{x} \sin \theta\right.\right. \\
& \left.\left.-u_{y} \cos \theta\right) \Delta\right] \times \exp \left[-2 \pi^{2}\left(u_{x}^{2}+u_{y}^{2}\right) \phi_{\text {blur }}^{2}\right]
\end{aligned}
$$

\section{ACKNOWLEDGMENTS}

This work is supported by the Thirty Meter Telescope (TMT) project. The authors gratefully acknowledge the support of the TMT partner institutions. They are the Association of Canadian Universities for Research in Astronomy (ACURA), the California Institute of Technology, and the University of California. This work was sup- ported as well by the Gordon and Betty Moore Foundation, the Canada Foundation for Innovation, the Ontario Ministry of Research and Innovation, the National Research Council of Canada, the Natural Sciences and Engineering Research Council of Canada (NSERC), the British Columbia Knowledge Development Fund, the Association of Universities for Research in Astronomy (AURA), and the U.S. National Science Foundation (NSF).

\section{REFERENCES}

1. P. L. Wizinowich, D. Le Mignant, A. H. Bouchez, R. D. Campbell, J. C. Y. Chin, A. R. Contos, M. A. van Dam, S. K. Hartman, E. M. Johansson, R. E. Lafon, H. Lewis, P. J. Stomski, D. M. Summers, C. G. Brown, P. M. Danforth, C. E. Max, and D. M. Pennington, "The W. M. Keck observatory laser guide star adaptive optics system: overview," Psychol. Forsch. 118, 297-309 (2006).

2. M. Boccas, F. Rigaut, M. Bec, B. Irarrazaval, E. James, A. Ebbers, C. d'Orgeville, K. Grace, G. Arriagada, S. Karewicz, M. Sheehan, J. White, and S. Chan, "Laser guide star upgrade of Altair at Gemini North," Proc. SPIE 6272, 62723L (2006).

3. M. Le Louarn, N. N. Hubin, and R. Arsenault, "Adaptive optics for second-generation VLT instruments," Proc. SPIE 5490, 248-259 (2004).

4. B. Ellerbroek, S. Adkins, D. Andersen, J. Atwood, C. Boyer, P. Byrnes, R. Conan, L. Gilles, G. Herriot, P. Hickson, E. Hileman, D. Joyce, B. Leckie, M. Liang, T. Pfrommer, J.-C. Sinquin, J.-P. Veran, L. Wang, and P. Welle, "Progress toward developing the TMT adaptive optical systems and their components," Proc. SPIE 7015, 70150R (2008).

5. E. Diolaiti, J.-M. Conan, I. Foppiani, M. Lombini, C. Petit, C. Robert, L. Schreiber, P. Ciliegi, E. Marchetti, M. Bellazzini, L. Busoni, S. Esposito, T. Fusco, N. Hubin, F. Quiros-Pacheco, A. Baruffolo, S. D’Odorico, J. Farinato, B. Neichel, R. Ragazzoni, C. Arcidiacono, V. Biliotti, G. Bregoli, G. Cosentino, and G. Innocenti, "A preliminary overview of the multiconjugate adaptive optics module for the E-ELT," Proc. SPIE 7015, 70150U (2008).

6. H. C. V. de Hulst, Light Scattering by Small Particles (Wiley, 1957)

7. E. J. McCartney, Optics of the Atmosphere: Scattering by Molecules and Particles (1976).

8. A. Bucholtz, "Rayleigh-scattering calculations for the terrestrial atmosphere," Appl. Opt. 34, 2765-2773 (1995).

9. S. S. Srivastava, N. K. Vyas, J. Rai, and B. Kartikeyan, "On the different approaches of Rayleigh optical depth determination," Adv. Space Res. 44, 1058-1066 (2009).

10. U.S. Standard Atmosphere Supplement (U.S. Government Print Office, 1966).

11. J. H. Seinfeld and S. N. Pandis, Atmospheric Chemistry and Physics-From Air Pollution to Climate Change (Wiley, 2006).

12. R. J. Sica, Z. A. Zylawy, and P. S. Argall, "Ozone corrections for Rayleigh-scatter temperature determinations in the middle atmosphere," J. Atmos. Oceanic Technol. 18-7, 1223-1228 (2001).

13. A. T. Young, http://mintaka.sdsu.edu/GF/explain/extinction/ extintro.html (2008).

14. Gemini, http://sciopsedit.gemini.edu/sciops/telescopes-andsites/observing-condition-constraints/extinction (2009).

15. G. E. Shaw, "Aerosols at Mauna Loa: optical properties," J. Atmos. Sci. 36, 862-869 (1979).

16. T. I. G. Eguchi and Nawo Yokota, "Characteristics of cirrus clouds from icesat/glas observations," Geophys. Res. Lett. 34, L09810 (2007).

17. L. Gilles and B. L. Ellerbroek, "Constrained matched filtering for extended dynamic range and improved noise rejection for Shack-Hartmann wavefront sensing," Opt. Lett. 33, 1159-1161 (2008). 